\title{
Article
}

\section{Nonlinear Pantograph-Type Diffusion PDEs: Exact Solutions and the Principle of Analogy}

\author{
Andrei D. Polyanin *(D) and Vsevolod G. Sorokin *(D) \\ Ishlinsky Institute for Problems in Mechanics RAS, 101 Vernadsky Avenue, bldg 1, 119526 Moscow, Russia \\ * Correspondence: polyanin@ipmnet.ru (A.D.P.); vsesor@gmail.com (V.G.S.)
}

check for

updates

Citation: Polyanin, A,D.; Sorokin, V.G. Nonlinear Pantograph-Type Diffusion PDEs: Exact Solutions and the Principle of Analogy. Mathematics 2021, 9, 511. https://doi.org/ $10.3390 /$ math 9050511

Academic Editor: Manuel de León

Received: 10 February 2021

Accepted: 24 February 2021

Published: 2 March 2021

Publisher's Note: MDPI stays neutral with regard to jurisdictional claims in published maps and institutional affiliations.

Copyright: (C) 2021 by the authors Licensee MDPI, Basel, Switzerland. This article is an open access article distributed under the terms and conditions of the Creative Commons Attribution (CC BY) license (https:/ / creativecommons.org/licenses/by/ $4.0 /)$.

\begin{abstract}
We study nonlinear pantograph-type reaction-diffusion PDEs, which, in addition to the unknown $u=u(x, t)$, also contain the same functions with dilated or contracted arguments of the form $w=u(p x, t), w=u(x, q t)$, and $w=u(p x, q t)$, where $p$ and $q$ are the free scaling parameters (for equations with proportional delay we have $0<p<1,0<q<1$ ). A brief review of publications on pantograph-type ODEs and PDEs and their applications is given. Exact solutions of various types of such nonlinear partial functional differential equations are described for the first time. We present examples of nonlinear pantograph-type PDEs with proportional delay, which admit traveling-wave and self-similar solutions (note that PDEs with constant delay do not have self-similar solutions). Additive, multiplicative and functional separable solutions, as well as some other exact solutions are also obtained. Special attention is paid to nonlinear pantograph-type PDEs of a rather general form, which contain one or two arbitrary functions. In total, more than forty nonlinear pantographtype reaction-diffusion PDEs with dilated or contracted arguments, admitting exact solutions, have been considered. Multi-pantograph nonlinear PDEs are also discussed. The principle of analogy is formulated, which makes it possible to efficiently construct exact solutions of nonlinear pantographtype PDEs. A number of exact solutions of more complex nonlinear functional differential equations with varying delay, which arbitrarily depends on time or spatial coordinate, are also described. The presented equations and their exact solutions can be used to formulate test problems designed to evaluate the accuracy of numerical and approximate analytical methods for solving the corresponding nonlinear initial-boundary value problems for PDEs with varying delay. The principle of analogy allows finding solutions to other nonlinear pantograph-type PDEs (including nonlinear wave-type PDEs and higher-order equations).
\end{abstract}

Keywords: nonlinear reaction-diffusion equations; pantograph-type differential equations; PDEs with proportional delay; PDEs with varying delay; partial functional-differential equations; exact solutions; self-similar solutions; additive and multiplicative separable solutions; functional separable solutions; generalized separable solutions

\section{Contents}

1. Introduction

1.1. Differential Equations with Constant Delay

1.2. Pantograph-Type ODEs and PDEs and Their Applications

1.3. Concept of 'Exact Solution' for Nonlinear Pantograph-Type PDEs

2. Solutions of Pantograph-Type PDEs That Contain Unknown Functions $u=u(x, t)$ and $w=u(p x, q t)$

2.1. Equations Containing Free Parameters

2.2. Equations Linear in Derivatives and Containing One Arbitrary Function

2.3. More Complex Nonlinear Equations Containing One Arbitrary Function

2.4. Nonlinear Equations Containing Two Arbitrary Functions 
3. Solutions of Pantograph-Type PDEs That Contain Unknown Functions $u=u(x, t)$ and $w=u(x, q t)$

3.1. Equations Containing Free Parameters

3.2. Equations Linear in Derivatives and Containing One Arbitrary Function

3.3. More Complex Nonlinear Equations Containing One Arbitrary Function

3.4. Nonlinear Equations Containing Two Arbitrary Functions

4. Solutions of Pantograph-Type PDEs That Contain Unknown Functions $u=u(x, t)$ and $w=u(p x, t)$

4.1. Equations Linear in Derivatives

4.2. More Complex Nonlinear Equations

5. Some Generalizations

5.1. Nonlinear Multi-Pantograph Type PDEs

5.2. Nonlinear Pantograph-Type PDEs Containing Unknown Functions with Dilation or Contraction of Arguments in the Derivative

5.3. Principle of Analogy of Solutions

6. Brief Conclusions

References

\section{Introduction}

\subsection{Differential Equations with Constant Delay}

In natural science, an important role is played by the study of the hereditary properties of nonlinear systems of different nature, when the rate of change of the unknown depends not only on the state at a given time, but also on the previous evolution of the process. In a particular case, the state of the system is determined not by its entire history, but by the current moment and some moment in the past. Such systems are called delay systems. In the simplest case, the differential equations modelling such systems, in addition to the unknown function $u(t)$, also contain the function $u(t-\tau)$, where $t$ is the time and $\tau>0$ is the delay [1-3]. The delay is generally assumed constant.

When modeling nonlinear systems with constant delay and two independent variables $x$ and $t$, where $x$ is the spatial coordinate, reaction-diffusion equations of the following form are most often encountered [4]:

$$
u_{t}=a u_{x x}+F(u, w), \quad w=u(x, t-\tau) .
$$

The presence of delay significantly complicates the analysis of equations of the form (1). Such equations admit traveling-wave solutions $u=u(z)$, where $z=x+\lambda t$ (e.g, see [4-7]), but do not admit self-similar solutions $u=t^{\beta} \varphi\left(x t^{\lambda}\right)$ (recall that many PDEs without delay have self-similar solutions).

More complex than traveling-wave, exact solutions of nonlinear delay reactiondiffusion equations are obtained in [8-19]. Exact solutions of nonlinear delay equations of the Klein-Gordon type and other nonlinear hyperbolic equations are given in [18-24]. Some exact solutions of differential-difference equations of a viscous incompressible fluid (which generalize the Navier-Stokes equations) are described in [25]. Numerical methods for solving delay PDEs are discussed, for example, in [26-28].

It is important to note that delay differential equations have a number of specific qualitative features $[4,21,29]$ that are not inherent in equations without delay.

\subsection{Pantograph-Type ODEs and PDEs and Their Applications}

In some cases, the delay may be time dependent, i.e., $\tau=\tau(t)$, where $\tau(t)>0$ is a given function [2].

To illustrate this, consider a first-order linear ODE with varying delay proportional to the independent variable:

$$
u_{t}^{\prime}=a u+b w, \quad w=u(p t)
$$


which for $p>0(p \neq 1)$ is called the pantograph equation.

For $0<p<1$, the equation describes the dynamics of a current collection system for an electric locomotive [30] and is a special case of an ODE with varying delay for $\tau(t)=(1-p) t$. The function $u(p t)$ included in the pantograph Equation (2) differs from the function $u(t)$ by dilation along the $t$-axis by $1 / p$ times.

The pantograph equation and more complex related ODEs and PDEs that contain the unknown functions with dilation (for $0<p<1$ ) or contraction (for $p>1$ ) of arguments are used in mathematical modeling of various processes in biology [31-35], astrophysics [36], electrodynamics [37], population theory [38], number theory [39], stochastic games [40], graph theory [41], risk and queue theory [42], and theory of neural networks [43]. Note that in [31,34-36] the equations are derived for the case $p>1$.

Analysis of pantograph-type ODEs and construction of approximate analytical solutions are carried out, for example, in [30,44-55]. Numerical methods for solving such equations are discussed in [56-81]. Thus, in general, approximate analytical and numerical methods for solving pantograph-type ODEs can currently be considered fairly well developed.

Significantly fewer publications are devoted to the analysis and solving of pantographtype PDEs. In [34], a linear first-order pantograph-type PDE is used to model the growth and division of cells structured by size. Its solution is sought in the form of a series, the terms of which are determined by solving simpler equations without dilated arguments. In [35], a more complex linear pantograph-type reaction-diffusion equation is investigated (obtained by adding a diffusion term to the equation that was analyzed in [34]). In [82], solutions of linear pantograph-type heat and wave equations are obtained using the method of separation of variables. In [83-85], the problems of unique solvability and smoothness of linear boundary value problems for elliptic PDEs with dilation or contraction of arguments in higher derivatives are considered. Analytical methods for solving some linear and nonlinear PDEs with proportional delays are discussed in [86-88]. In [89], a finite-difference scheme for the numerical integration of first-order PDEs with constant delay in $t$ and proportional delay in $x$ is constructed. Papers [90-92] are devoted to numerical methods for solving pantograph-type PDEs with proportional delay $[90,91]$ and more complex varying delay [92].

This article describes different classes of exact solutions to nonlinear pantograph-type reaction-diffusion equations of the form

$$
u_{t}=\left[g(u) u_{x}\right]_{x}+F(u, w)
$$

where $u=u(x, t)$ and $w=u(p x, q t), p>0$ and $q>0$ ( $p$ and $q$ cannot be equal to 1 at the same time).

Remark 1. Exact solutions of 'ordinary' nonlinear diffusion type equations (without dilated or contracted arguments) can be found, for example, in [93-117].

In most cases, exact solutions of pantograph-type PDEs of the form (3) are obtained as follows: a solution to a pantograph-type equation is sought in the same form as a solution to a simpler equation without delay, which is obtained from (3) for $w=u$ (for details, see Section 5.3). More complex exact solutions are found using various modifications of the functional constraints method $[10,15]$. In addition, to construct some exact solutions of pantograph-type equations, we use exact solutions of nonlinear PDEs with varying delay of general form, which were given in [9,11,15].

As a result of the analysis, we have managed to find many nonlinear pantograph-type reaction-diffusion equations, which admit exact traveling-wave solutions, self-similar solutions, additive, multiplicative, generalized, and functional separable solutions, as well as some other exact solutions. Special attention is paid to nonlinear pantograph-type PDEs of a rather general form that contain arbitrary functions (it is these equations and their solutions that are of greatest interest for testing numerical and approximate analytical 
methods for solving the corresponding nonlinear initial-boundary value problems for PDEs with varying delay).

\subsection{Concept of 'Exact Solution' for Nonlinear Pantograph-Type PDEs}

In this article, the term 'exact solution' for nonlinear pantograph-type PDEs (with dilation or contraction of one or several independent variables) and equations with varying delay will be used in cases where the solution is expressed:

(i) in terms of elementary functions, functions included in the equation (this is necessary when the equation contains arbitrary functions), and indefinite and/or definite integrals;

(ii) in terms of solutions of ODEs without delay or systems of such equations;

(iii) in terms of solutions of pantograph-type ODEs and ODEs with constant or varying delay or systems of such equations.

Combinations of solutions from paragraphs (i)-(iii) are allowed. In case (i), an exact solution can be represented in explicit, implicit or parametric form. This definition generalizes the concept of 'exact solution' that is used for nonlinear PDEs with constant delay in $[10,12]$ and for PDEs without delay in [93].

In what follows, unless otherwise specified, in nonlinear pantograph-type PDEs, it is assumed that $p$ and $q$ are the free scaling parameters ( $p$ and $q$ are positive and cannot be equal to 1 at the same time), $f(z)$ and $g(z)$ are arbitrary functions, and $a, b, c$, etc. are arbitrary real constants. The unknown function is denoted by $u$, and the unknown function with dilation or contraction of one or more arguments is denoted by $w$.

\section{Solutions of Pantograph-Type PDEs That Contain Unknown Functions $u=u(x, t)$ and $w=u(p x, q t)$}

2.1. Equations Containing Free Parameters

Equation 1. The equation with power-law nonlinearity

$$
u_{t}=a u_{x x}+b w^{k}, \quad w=u(p x, q t)
$$

for $k \neq 1$ admits the self-similar solution

$$
u(x, t)=t^{\frac{1}{1-k}} U(z), \quad z=x t^{-1 / 2}
$$

where the function $U=U(z)$ is described by the nonlinear pantograph-type ODE:

$$
a U_{z z}^{\prime \prime}+\frac{1}{2} z U_{z}^{\prime}-\frac{1}{1-k} U+b q^{\frac{k}{1-k}} W^{k}=0, \quad W=U(s z), \quad s=p q^{-1 / 2} .
$$

Remark 2. It is interesting to note that the ODE with proportional delay (4) for $0<p, q<1$ in the special case $p=q^{1 / 2}$ has an exact solution expressed in terms of the solution of the ODE without delay (6) with $s=1$; for $p<q^{1 / 2}$, Equation (4) reduces to the delay $O D E$ with $s<1$; and for $p>q^{1 / 2}$, to the ODE with contracted argument with $s>1$. Moreover, a solution of the ODE (4) for $p, q>1$ for appropriate values of the parameters $p$ and $q$ can also be expressed in terms of the solution of the ODE with delay $(s<1)$, without delay $(s=1)$, and with contracted argument ( $s>1$ ).

The equations and their solutions given below in Section 2 have similar qualitative features.

Equation 2. The more complex equation with power-law nonlinearity

$$
u_{t}=a u_{x x}+b u^{m} w^{k}, \quad w=u(p x, q t),
$$

for $k \neq 1-m$ admits the self-similar solution

$$
u(x, t)=t^{\frac{1}{1-m-k}} U(z), \quad z=x t^{-1 / 2},
$$


where the function $U=U(z)$ is described by the nonlinear pantograph-type ODE:

$$
\begin{aligned}
& a U_{z z}^{\prime \prime}+\frac{1}{2} z U_{z}^{\prime}-\frac{1}{1-m-k} U+b q^{\frac{k}{1-m-k}} U^{m} W^{k}=0, \\
& W=U(s z), \quad s=p q^{-1 / 2} .
\end{aligned}
$$

Equation 3. The equation with exponential nonlinearity

$$
u_{t}=a u_{x x}+b e^{\lambda w}, \quad w=u(p x, q t),
$$

admits the exact solution

$$
u(x, t)=U(z)-\frac{1}{\lambda} \ln t, \quad z=x t^{-1 / 2},
$$

where the function $U=U(z)$ is described by the nonlinear pantograph-type ODE:

$$
\begin{aligned}
& a U_{z z}^{\prime \prime}+\frac{1}{2} z U_{z}^{\prime}+\frac{1}{\lambda}+\frac{b}{q} e^{\lambda W}=0, \\
& W=U(s z), \quad s=p q^{-1 / 2} .
\end{aligned}
$$

Equation 4. The more complex equation with exponential nonlinearity

$$
u_{t}=a u_{x x}+b e^{\mu u+\lambda w}, \quad w=u(p x, q t),
$$

for $\mu \neq-\lambda$ admits the exact solution

$$
u(x, t)=U(z)-\frac{1}{\mu+\lambda} \ln t, \quad z=x t^{-1 / 2},
$$

where the function $U=U(z)$ is described by the nonlinear pantograph-type ODE:

$$
\begin{aligned}
& a U_{z z}^{\prime \prime}+\frac{1}{2} z U_{z}^{\prime}+\frac{1}{\mu+\lambda}+b q^{-\frac{\lambda}{\mu+\lambda}} e^{\mu U+\lambda W}=0, \\
& W=U(s z), \quad s=p q^{-1 / 2} .
\end{aligned}
$$

Equation 5. The equation with logarithmic nonlinearity

$$
u_{t}=a u_{x x}+u(b \ln u+c \ln w+d), \quad w=u(p x, q t),
$$

admits the multiplicative separable solution

$$
u(x, t)=\varphi(x) \psi(t),
$$

where the functions $\varphi=\varphi(x)$ and $\psi=\psi(t)$ are described by the nonlinear pantographtype ODEs:

$$
\begin{aligned}
& a \varphi_{x x}^{\prime \prime}+\varphi(b \ln \varphi+c \ln \bar{\varphi}+d)=0, \quad \bar{\varphi}=\varphi(p x) \\
& \psi_{t}^{\prime}=\psi(b \ln \psi+c \ln \bar{\psi}), \quad \bar{\psi}=\psi(q t) .
\end{aligned}
$$

Remark 3. Equation (7) and its solution can be substantially generalized if the pantograph term $w=u(p x, q t)$ is replaced by $w=u(\xi(x), \eta(t))$, where $\xi(x)$ and $\eta(t)$ are arbitrary functions (the solution is sought in the same form (8)). 


\subsection{Equations Linear in Derivatives and Containing One Arbitrary Function}

Equation 6. The equation containing an arbitrary function

$$
u_{t}=a u_{x x}+u^{n} f(w / u), \quad w=u(p x, q t),
$$

for $n \neq 1$ admits the self-similar solution

$$
u(x, t)=t^{\frac{1}{1-n}} U(z), \quad z=x t^{-1 / 2},
$$

where the function $U=U(z)$ satisfies the second-order pantograph-type ODE:

$$
\begin{aligned}
& \frac{1}{1-n} U-\frac{1}{2} z U_{z}^{\prime}=a U_{z z}^{\prime \prime}+U^{n} f\left(q^{\frac{1}{1-n}} W / U\right), \\
& W=U(s z), \quad s=p q^{-1 / 2} .
\end{aligned}
$$

Equation 7. The equation containing an arbitrary function

$$
u_{t}=a u_{x x}+e^{\lambda u} f(u-w), \quad w=u(p x, q t),
$$

admits the exact solution

$$
u(x, t)=U(z)-\frac{1}{\lambda} \ln t, \quad z=x t^{-1 / 2},
$$

where the function $U=U(z)$ is described by the nonlinear pantograph-type ODE:

$$
\begin{aligned}
& a U_{z z}^{\prime \prime}+\frac{1}{2} z U_{z}^{\prime}+\frac{1}{\lambda}+e^{\lambda U} f\left(U-W+\frac{1}{\lambda} \ln q\right)=0, \\
& W=U(s z), \quad s=p q^{-1 / 2} .
\end{aligned}
$$

\subsection{More Complex Nonlinear Equations Containing One Arbitrary Function}

Equation 8 . The equation with varying transfer coefficient of power-law type

$$
u_{t}=a\left(u^{k} u_{x}\right)_{x}+u^{n} f(w / u), \quad w=u(p x, q t),
$$

for $n \neq 1$ admits the self-similar solution

$$
u(x, t)=t^{\frac{1}{1-n}} U(z), \quad z=x t^{\frac{n-k-1}{2(1-n)}},
$$

where the function $U=U(z)$ satisfies the second-order pantograph-type ODE:

$$
\begin{aligned}
& \frac{1}{1-n} U+\frac{n-k-1}{2(1-n)} z U_{z}^{\prime}=a\left(U^{k} U_{z}^{\prime}\right)_{z}^{\prime}+U^{n} f\left(q^{\frac{1}{1-n}} W / U\right), \\
& W=U(s z), \quad s=p q^{\frac{n-k-1}{2(1-n)}} .
\end{aligned}
$$

Equation 9. The equation containing an arbitrary function

$$
u_{t}=a\left(e^{\lambda u} u_{x}\right)_{x}+e^{\mu u} f(u-w), \quad w=u(p x, q t),
$$

for $\mu \neq 0$ admits the exact solution

$$
u(x, t)=U(z)-\frac{1}{\mu} \ln t, \quad z=x t^{\frac{\lambda-\mu}{2 \mu}},
$$


where the function $U=U(z)$ is described by the nonlinear pantograph-type ODE:

$$
\begin{aligned}
& \frac{\lambda-\mu}{2 \mu} z U_{z}^{\prime}-\frac{1}{\mu}=a\left(e^{\lambda U} U_{z}^{\prime}\right)_{z}^{\prime}+e^{\mu U} f\left(U-W+\frac{1}{\mu} \ln q\right)=0, \\
& W=U(s z), \quad s=p q^{\frac{\lambda-\mu}{2 \mu}} .
\end{aligned}
$$

\subsection{Nonlinear Equations Containing Two Arbitrary Functions}

Equation 10. The equation with varying transfer coefficient of general form

$$
u_{t}=a\left[u f_{u}^{\prime}(u) u_{x}\right]_{x}+\frac{1}{f_{u}^{\prime}(u)}[b f(u)+c f(w)+d], \quad w=u(p x, q t),
$$

admits the functional separable solution in implicit form

$$
f(u)=\varphi(t) x+\psi(t),
$$

where the functions $\varphi=\varphi(t)$ and $\psi=\psi(t)$ satisfy the pantograph-type ODEs:

$$
\begin{aligned}
& \varphi_{t}^{\prime}=b \varphi+c p \bar{\varphi}, \quad \bar{\varphi}=\varphi(q t), \\
& \psi_{t}^{\prime}=b \psi+c \bar{\psi}+d+a \varphi^{2}, \quad \bar{\psi}=\psi(q t) .
\end{aligned}
$$

Remark 4. More general than Equation (11), the equation in which the pantograph term $w=$ $u(p x, q t)$ is replaced by $w=u\left(p_{1} x+p_{0}, q_{1} t+q_{0}\right)$ also has the functional separable solution (12). The more complex functional differential equation in which $w=u\left(p_{1} x+p_{0}, \eta(t)\right)$, where $\eta(t)$ is an arbitrary function, also has a solution of the same form.

Equation 11. For $q=p$, a nonlinear pantograph-type reaction-diffusion equation of the general form

$$
u_{t}=\left[g(u) u_{x}\right]_{x}+F(u, w), \quad w=u(p x, p t),
$$

admits the traveling-wave solution

$$
u(x, t)=U(z), \quad z=k x-\lambda t,
$$

where $k$ and $\lambda$ are arbitrary constants and the function $U=U(z)$ is described by the nonlinear pantograph-type ODE:

$$
k^{2}\left[g(U) U_{z}^{\prime}\right]_{z}^{\prime}+\lambda U_{z}^{\prime}+F(U, W)=0, \quad W=U(p z) .
$$

3. Solutions of Pantograph-Type PDEs That Contain Unknown Functions $u=u(x, t)$ and $w=u(x, q t)$

This section does not include equations and their solutions that can be obtained by setting $p=1$ in the equations and solutions considered in Section 2.

\subsection{Equations Containing Free Parameters}

Equation 12. The equation with logarithmic nonlinearity

$$
u_{t}=a u_{x x}+u(b \ln u+c \ln w+d), \quad w=u(x, q t),
$$

admits the functional separable solution

$$
u(x, t)=\exp \left[\psi_{2}(t) x^{2}+\psi_{1}(t) x+\psi_{0}(t)\right]
$$


where the functions $\psi_{n}=\psi_{n}(t)$ are described by the nonlinear system of pantographtype ODEs:

$$
\begin{aligned}
& \psi_{2}^{\prime}=4 a \psi_{2}^{2}+b \psi_{2}+c \bar{\psi}_{2}, \quad \bar{\psi}_{2}=\psi_{2}(q t), \\
& \psi_{1}^{\prime}=4 a \psi_{1} \psi_{2}+b \psi_{1}+c \bar{\psi}_{1}, \quad \bar{\psi}_{1}=\psi_{1}(q t), \\
& \psi_{0}^{\prime}=a\left[\psi_{1}^{2}+2 \psi_{2}\right]+b \psi_{0}+c \bar{\psi}_{0}+d, \quad \bar{\psi}_{0}=\psi_{0}(q t) .
\end{aligned}
$$

Equation 13. The equation with logarithmic nonlinearity

$$
u_{t}=a u_{x x}+u\left(b \ln ^{2} u+c \ln u+d \ln w+s\right), \quad w=u(x, q t),
$$

depending on the sign of the product $a b$, admits two functional separable solutions given below.

$1^{\circ}$. The solution for $a b>0$ :

$$
\begin{aligned}
& u(x, t)=\exp \left[\psi_{1}(t) \varphi(x)+\psi_{2}(t)\right], \\
& \varphi(x)=A \cos (\lambda x)+B \sin (\lambda x), \quad \lambda=\sqrt{b / a},
\end{aligned}
$$

where $A$ and $B$ are arbitrary constants and the functions $\psi_{n}=\psi_{n}(t)$ are described by the nonlinear system of pantograph-type ODEs:

$$
\begin{aligned}
& \psi_{1}^{\prime}=2 b \psi_{1} \psi_{2}+(c-b) \psi_{1}+d \bar{\psi}_{1}, \quad \bar{\psi}_{1}=\psi_{1}(q t), \\
& \psi_{2}^{\prime}=b\left(A^{2}+B^{2}\right) \psi_{1}^{2}+b \psi_{2}^{2}+c \psi_{2}+d \bar{\psi}_{2}+s, \quad \bar{\psi}_{2}=\psi_{2}(q t) .
\end{aligned}
$$

$2^{\circ}$. The solution for $a b<0$ :

$$
\begin{aligned}
& u(x, t)=\exp \left[\psi_{1}(t) \varphi(x)+\psi_{2}(t)\right], \\
& \varphi(x)=A \cosh (\lambda x)+B \sinh (\lambda x), \quad \lambda=\sqrt{-b / a},
\end{aligned}
$$

where $A$ and $B$ are arbitrary constants and the functions $\psi_{n}=\psi_{n}(t)$ are described by the nonlinear system of pantograph-type ODEs:

$$
\begin{aligned}
& \psi_{1}^{\prime}=2 b \psi_{1} \psi_{2}+(c-b) \psi_{1}+d \bar{\psi}_{1}, \quad \bar{\psi}_{1}=\psi_{1}(q t), \\
& \psi_{2}^{\prime}=b\left(A^{2}-B^{2}\right) \psi_{1}^{2}+b \psi_{2}^{2}+c \psi_{2}+d \bar{\psi}_{2}+s, \quad \bar{\psi}_{2}=\psi_{2}(q t) .
\end{aligned}
$$

For $A= \pm B$, we have $\varphi(x)=A e^{ \pm \lambda x}$. In this case, the second equation of the system becomes independent and the first one becomes linear in $\psi_{1}$.

Remark 5. Equations (13) and (14) and their solutions can be substantially generalized if the pantograph term $w=u(x, q t)$ is replaced by $w=u(x, \eta(t))$, where $\eta(t)$ is an arbitrary function (the solutions are sought in the same form, see [9]).

\subsection{Equations Linear in Derivatives and Containing One Arbitrary Function}

Equation 14. The equation containing an arbitrary function

$$
u_{t}=a u_{x x}+f(u-w), \quad w=u(x, q t),
$$

admits the additive separable solution

$$
u(x, t)=C_{1} x^{2}+C_{2} x+\psi(t),
$$

where $C_{1}$ and $C_{2}$ are arbitrary real constants and the function $\psi=\psi(t)$ is described by the nonlinear first-order pantograph-type ODE:

$$
\psi_{t}^{\prime}=2 a C_{1}+f(\psi-\bar{\psi}), \quad \bar{\psi}=\psi(q t) .
$$


Equation 15. The equation containing an arbitrary function

$$
u_{t}=a u_{x x}+b u+f(u-w), \quad w=u(x, q t),
$$

depending on the sign of the product $a b$, admits two additive separable solutions given below.

$1^{\circ}$. The solution for $a b<0$ :

$$
u(x, t)=A \cosh (\lambda x)+B \sinh (\lambda x)+\psi(t), \quad \lambda=\sqrt{-b / a},
$$

where $A$ and $B$ are arbitrary constants and the function $\psi=\psi(t)$ is described by the nonlinear first-order pantograph-type ODE:

$$
\psi_{t}^{\prime}=b \psi+f(\psi-\bar{\psi}), \quad \bar{\psi}=\psi(q t) .
$$

$2^{\circ}$. The solution for $a b>0$ :

$$
u(x, t)=A \cos (\lambda x)+B \sin (\lambda x)+\psi(t), \quad \lambda=\sqrt{b / a},
$$

where $A$ and $B$ are arbitrary constants and the function $\psi=\psi(t)$ is described by the nonlinear first-order pantograph-type ODE (16).

Note that Equation (15) and its solutions can be substantially generalized if the pantograph term $w=u(x, q t)$ is replaced by $w=u(x, \eta(t))$, where $\eta(t)$ is an arbitrary function (the solutions are sought in the same form, see $[9,11])$.

Equation 16. The equation containing an arbitrary function

$$
u_{t}=a u_{x x}+u f(w / u), \quad w=u(x, q t),
$$

admits several multiplicative separable solutions given below.

$1^{\circ}$. The solution

$$
u(x, t)=[A \cosh (\lambda x)+B \sinh (\lambda x)] \psi(t),
$$

where $A, B$, and $\lambda$ are arbitrary constants and the function $\psi=\psi(t)$ is described by the nonlinear first-order pantograph-type ODE:

$$
\psi_{t}^{\prime}=a \lambda^{2} \psi+\psi f(\bar{\psi} / \psi), \quad \bar{\psi}=\psi(q t) .
$$

$2^{\circ}$. The solution

$$
u(x, t)=[A \cos (\lambda x)+B \sin (\lambda x)] \psi(t),
$$

where $A, B$, and $\lambda$ are arbitrary constants and the function $\psi=\psi(t)$ is described by the nonlinear first-order pantograph-type ODE:

$$
\psi_{t}^{\prime}=-a \lambda^{2} \psi+\psi f(\bar{\psi} / \psi), \quad \bar{\psi}=\psi(q t) .
$$

$3^{\circ}$. The degenerate solution

$$
u(x, t)=(A x+B) \psi(t),
$$

where $A, B$, and $\lambda$ are arbitrary constants and the function $\psi=\psi(t)$ is described by the nonlinear first-order pantograph-type ODE:

$$
\psi_{t}^{\prime}=\psi f(\bar{\psi} / \psi), \quad \bar{\psi}=\psi(q t) .
$$

Equation 17. The equation containing an arbitrary function

$$
u_{t}=a u_{x x}+b u \ln u+u f(w / u), \quad w=u(x, q t),
$$


admits the multiplicative separable solution

$$
u(x, t)=\varphi(x) \psi(t) .
$$

where the functions $\varphi=\varphi(x)$ and $\psi=\psi(t)$ are described by the nonlinear second-order ODE and the nonlinear first-order pantograph-type ODE:

$$
\begin{aligned}
& a \varphi_{x x}^{\prime \prime}=C_{1} \varphi-b \varphi \ln \varphi, \\
& \psi_{t}^{\prime}=C_{1} \psi+\psi f(\bar{\psi} / \psi)+b \psi \ln \psi, \quad \bar{\psi}=\psi(q t) ;
\end{aligned}
$$

$C_{1}$ is an arbitrary constant.

The first equation of Equation (18) is autonomous; its general solution can be obtained in implicit form. A particular one-parameter solution of this equation has the form

$$
\varphi=\exp \left[-\frac{b}{4 a}\left(x+C_{2}\right)^{2}+\frac{C_{1}}{b}+\frac{1}{2}\right],
$$

where $C_{2}$ is an arbitrary constant.

Note that Equation (17) and its solution can be substantially generalized if the pantograph term $w=u(x, q t)$ is replaced by $w=u(x, \eta(t))$, where $\eta(t)$ is an arbitrary function (the solution is sought in the same form, see $[9,11]$ ).

\subsection{More Complex Nonlinear Equations Containing One Arbitrary Function}

Equation 18. The equation with varying transfer coefficient of power-law type

$$
u_{t}=a\left(u^{k} u_{x}\right)_{x}+u f(w / u), \quad w=u(x, q t)
$$

admits the multiplicative separable solution

$$
u(x, t)=\varphi(x) \psi(t),
$$

where the functions $\varphi=\varphi(x)$ and $\psi=\psi(t)$ are determined from the ODE and the pantograph-type ODE:

$$
\begin{aligned}
& a\left(\varphi^{k} \varphi_{x}^{\prime}\right)_{x}^{\prime}=b \varphi, \\
& \psi_{t}^{\prime}=b \psi^{k+1}+\psi f(\bar{\psi} / \psi), \quad \bar{\psi}=\psi(q t) ;
\end{aligned}
$$

$b$ is an arbitrary constant.

Equation 19. The equation with varying transfer coefficient of power-law type

$$
u_{t}=a\left(u^{k} u_{x}\right)_{x}+u f(w / u)+b u^{k+1}, \quad w=u(x, q t),
$$

admits three multiplicative separable solutions given below.

$1^{\circ}$. The solution for $b(k+1)>0$ :

$$
u(x, t)=\left[C_{1} \cos (\beta x)+C_{2} \sin (\beta x)\right]^{1 /(k+1)} \psi(t), \quad \beta=\sqrt{b(k+1) / a},
$$

where $C_{1}$ and $C_{2}$ are arbitrary constants and the function $\psi=\psi(t)$ is described by the pantograph-type ODE:

$$
\psi_{t}^{\prime}=\psi f(\bar{\psi} / \psi), \quad \bar{\psi}=\psi(q t) .
$$

$2^{\circ}$. The solution for $b(k+1)<0$ :

$$
u(x, t)=\left[C_{1} \exp (-\beta x)+C_{2} \exp (\beta x)\right]^{1 /(k+1)} \psi(t), \quad \beta=\sqrt{-b(k+1) / a},
$$

where $C_{1}$ and $C_{2}$ are arbitrary constants and the function $\psi=\psi(t)$ is described by the pantograph-type ODE (21). 
$3^{\circ}$. The solution for $k=-1$ :

$$
u(x, t)=C_{1} \exp \left(-\frac{b}{2 a} x^{2}+C_{2} x\right) \psi(t),
$$

where $C_{1}$ and $C_{2}$ are arbitrary constants and the function $\psi=\psi(t)$ is described by the pantograph-type ODE (21).

Remark 6. Equations (19) and (20) and their solutions can be substantially generalized if the pantograph term $w=u(x, q t)$ is replaced by $w=u(x, \eta(t))$, where $\eta(t)$ is an arbitrary function (the solutions are sought in the same form, see [15]).

Equation 20. The equation with varying transfer coefficient of power-law type

$$
u_{t}=a\left(u^{k} u_{x}\right)_{x}+u^{k+1} f(w / u), \quad w=u(x, q t),
$$

admits the exact solution

$$
u(x, t)=t^{-1 / k} \varphi(z), \quad z=x+\lambda \ln t,
$$

where $\lambda$ is an arbitrary constant and the function $\varphi=\varphi(z)$ satisfies the second-order ODE with constant delay

$$
a\left(\varphi^{k} \varphi_{z}^{\prime}\right)_{z}^{\prime}-\lambda \varphi_{z}^{\prime}+\frac{1}{k} \varphi+\varphi^{k+1} f\left(q^{-1 / k} \bar{\varphi} / \varphi\right)=0, \quad \bar{\varphi}=\varphi(z+\lambda \ln q) .
$$

Equation 21. The equation with varying transfer coefficient of power-law type

$$
u_{t}=a\left(u^{k} u_{x}\right)_{x}+b+u^{-k} f\left(u^{k+1}-w^{k+1}\right), \quad w=u(x, q t),
$$

admits the functional separable solution

$$
u(x, t)=\left[\psi(t)-\frac{b(k+1)}{2 a} x^{2}+C_{1} x+C_{2}\right]^{1 /(k+1)},
$$

where $C_{1}$ and $C_{2}$ are arbitrary constants and the function $\psi=\psi(t)$ is described by the pantograph-type ODE:

$$
\psi_{t}^{\prime}=(k+1) f(\psi-\bar{\psi}), \quad \bar{\psi}=\psi(q t) .
$$

Equation 22. The equation with varying transfer coefficient of exponential type

$$
u_{t}=a\left(e^{\lambda u} u_{x}\right)_{x}+f(u-w), \quad w=u(x, q t),
$$

admits the additive separable solution

$$
u(x, t)=\frac{1}{\lambda} \ln \left(A x^{2}+B x+C\right)+\psi(t),
$$

where $A, B$, and $C$ are arbitrary constants and the function $\psi(t)$ is described by the pantograph-type ODE:

$$
\psi^{\prime}=2 a(A / \lambda) e^{\lambda \psi}+f(\psi-\bar{\psi}), \quad \bar{\psi}=\psi(q t) .
$$

Equation 23. The equation with varying transfer coefficient of exponential type

$$
u_{t}=a\left(e^{\lambda u} u_{x}\right)_{x}+f(u-w)+b e^{\lambda u}, \quad w=u(x, q t),
$$

admits two additive separable solutions. 
$1^{\circ}$. The solution for $b \lambda>0$ :

$$
u(x, t)=\frac{1}{\lambda} \ln \left[C_{1} \cos (\beta x)+C_{2} \sin (\beta x)\right]+\psi(t), \quad \beta=\sqrt{b \lambda / a},
$$

where $C_{1}$ and $C_{2}$ are arbitrary constants and the function $\psi=\psi(t)$ is described by the pantograph-type ODE:

$$
\psi_{t}^{\prime}=f(\psi-\bar{\psi}), \quad \bar{\psi}=\psi(q t) .
$$

$2^{\circ}$. The solution for $b \lambda<0$ :

$$
u(x, t)=\frac{1}{\lambda} \ln \left[C_{1} \exp (-\beta x)+C_{2} \exp (\beta x)\right]+\psi(t), \quad \beta=\sqrt{-b \lambda / a},
$$

where $C_{1}$ and $C_{2}$ are arbitrary constants and the function $\psi=\psi(t)$ is described by the pantograph-type ODE (25).

Remark 7. Equations (22)-(24) and their solutions can be substantially generalized if the pantograph term $w=u(x, q t)$ is replaced by $w=u(x, \eta(t))$, where $\eta(t)$ is an arbitrary function (the solutions are sought in the same form, see [15]).

Equation 24. The equation with varying transfer coefficient of exponential type

$$
u_{t}=a\left(e^{\lambda u} u_{x}\right)_{x}+b+e^{-\lambda u} f\left(e^{\lambda u}-e^{\lambda w}\right), \quad w=u(x, q t)
$$

admits the functional separable solution

$$
u(x, t)=\frac{1}{\lambda} \ln \left[\psi(t)-\frac{b \lambda}{2 a} x^{2}+C_{1} x+C_{2}\right]
$$

where $C_{1}$ and $C_{2}$ are arbitrary constants and the function $\psi=\psi(t)$ is described by the pantograph-type ODE:

$$
\psi_{t}^{\prime}=\lambda f(\psi-\bar{\psi}), \quad \bar{\psi}=\psi(q t) .
$$

Equation 25. The equation with varying transfer coefficient of logarithmic type

$$
u_{t}=\left[(a \ln u+b) u_{x}\right]_{x}-c u \ln u+u f(w / u), \quad w=u(x, q t),
$$

admits two multiplicative separable solutions

$$
u(x, t)=\exp ( \pm \sqrt{c / a} x) \psi(t)
$$

where the function $\psi=\psi(t)$ is described by the pantograph-type ODE:

$$
\psi_{t}^{\prime}=c(1+b / a) \psi+\psi f(\bar{\psi} / \psi), \quad \bar{\psi}=\psi(q t) .
$$

\subsection{Nonlinear Equations Containing Two Arbitrary Functions}

Equation 26. The equation with varying transfer coefficient of general form

$$
u_{t}=a\left[g_{u}^{\prime}(u) u_{x}\right]_{x}+b+\frac{1}{g_{u}^{\prime}(u)} f(g(u)-g(w)), \quad w=u(x, q t),
$$

admits the functional separable solution in implicit form

$$
g(u)=\psi(t)-\frac{b}{2 a} x^{2}+C_{1} x+C_{2}
$$

where the function $\psi=\psi(t)$ is described by the pantograph-type ODE (25). 
Equation 27. The equation with varying transfer coefficient of general form

$$
u_{t}=a\left[g_{u}^{\prime}(u) u_{x}\right]_{x}+b g(u)+\frac{g(u)}{g_{u}^{\prime}(u)} f(g(w) / g(u)), \quad w=u(x, q t),
$$

admits two functional separable solutions in implicit form.

$1^{\circ}$. The solution for $a b>0$ :

$$
g(u)=\left[C_{1} \cos (\lambda x)+C_{2} \sin (\lambda x)\right] \psi(t), \quad \lambda=\sqrt{b / a},
$$

where $C_{1}$ and $C_{2}$ are arbitrary constants and the function $\psi=\psi(t)$ is described by the pantograph-type ODE (21).

$2^{\circ}$. The solution for $a b<0$ :

$$
g(u)=\left[C_{1} \exp (-\lambda x)+C_{2} \exp (\lambda x)\right] \psi(t), \quad \lambda=\sqrt{-b / a},
$$

where $C_{1}$ and $C_{2}$ are arbitrary constants and the function $\psi=\psi(t)$ is described by the pantograph-type ODE (21).

Remark 8. Equations (26)-(29) and their solutions can be substantially generalized if the pantograph term $w=u(x, q t)$ is replaced by $w=u(x, \eta(t))$, where $\eta(t)$ is an arbitrary function (the solutions are sought in the same form, see [15]).

\section{Solutions of Pantograph-Type PDEs That Contain Unknown Functions $u=u(x, t)$ and $w=u(p x, t)$}

Preliminary remarks. This section does not include equations and their solutions that can be obtained by setting $q=1$ in the equations and solutions considered in Section 2 .

\subsection{Equations Linear in Derivatives}

Equation 28. The equation containing an arbitrary function

$$
u_{t}=a u_{x x}+f(u-w), \quad w=u(p x, t),
$$

admits the additive separable solution

$$
u(x, t)=C t+\varphi(x),
$$

where $C$ is an arbitrary constant and the function $\varphi=\varphi(x)$ is described by the nonlinear second-order pantograph-type ODE:

$$
a \varphi_{x x}^{\prime \prime}-C+f(\varphi-\bar{\varphi})=0, \quad \bar{\varphi}=\varphi(p x) .
$$

Equation 29. The equation containing an arbitrary function

$$
u_{t}=a u_{x x}+b u+f(u-w), \quad w=u(p x, t),
$$

admits the additive separable solution

$$
u(x, t)=C e^{b t}+\varphi(x),
$$

where $C$ is an arbitrary constant and the function $\varphi=\varphi(x)$ is described by the nonlinear second-order pantograph-type ODE:

$$
a \varphi_{x x}^{\prime \prime}+b \varphi+f(\varphi-\bar{\varphi})=0, \quad \bar{\varphi}=\varphi(p x) .
$$

Equation 30. The equation containing an arbitrary function

$$
u_{t}=a u_{x x}+u f(w / u), \quad w=u(p x, t),
$$


admits the multiplicative separable solution

$$
u(x, t)=e^{\lambda t} \varphi(x),
$$

where $\lambda$ is an arbitrary constant and the function $\varphi=\varphi(x)$ is described by the nonlinear second-order pantograph-type ODE:

$$
a \varphi_{x x}^{\prime \prime}+\varphi[f(\bar{\varphi} / \varphi)-\lambda]=0, \quad \bar{\varphi}=\varphi(p x) .
$$

Equation 31. The equation containing an arbitrary function

$$
u_{t}=a u_{x x}+b u \ln u+u f(w / u), \quad w=u(p x, t),
$$

admits the multiplicative separable solution

$$
u(x, t)=\exp \left(C e^{b t}\right) \varphi(x),
$$

where $C$ is an arbitrary constant and the function $\varphi=\varphi(x)$ is described by the nonlinear pantograph-type ODE:

$$
a \varphi_{x x}^{\prime \prime}+b \varphi \ln \varphi+\varphi f(\bar{\varphi} / \varphi)=0, \quad \bar{\varphi}=\varphi(p x) .
$$

Remark 9. Equations (30)-(33) and their solutions can be substantially generalized if the pantograph term $w=u(p x, t)$ is replaced by $w=u(\xi(x), t)$, where $\xi(x)$ is an arbitrary function (the solutions are sought in the same form).

\subsection{More Complex Nonlinear Equations}

Equation 32. The equation with varying transfer coefficient of power-law type

$$
u_{t}=a\left(u^{k} u_{x}\right)_{x}+u f(w / u), \quad w=u(p x, t)
$$

admits the exact solution

$$
u(x, t)=e^{2 \lambda t} U(z), \quad z=e^{-k \lambda t} x,
$$

where $\lambda$ is an arbitrary constant and the function $U=U(z)$ is determined from the pantograph-type ODE:

$$
2 \lambda U-k \lambda z U_{z}^{\prime}=a\left(U^{k} U_{z}^{\prime}\right)_{z}^{\prime}+U f(W / U), \quad W=U(p z) .
$$

Equation 33. The equation with varying transfer coefficient of power-law type

$$
u_{t}=a\left(u^{k} u_{x}\right)_{x}+u^{k+1} f(w / u), \quad w=u(p x, t) .
$$

admits the multiplicative separable solution

$$
u(x, t)=t^{-1 / k} \varphi(x),
$$

where the function $\varphi=\varphi(x)$ is described by the pantograph-type ODE:

$$
a\left(\varphi^{k} \varphi_{x}^{\prime}\right)_{x}^{\prime}+\frac{1}{k} \varphi+\varphi^{k+1} f(\bar{\varphi} / \varphi)=0, \quad \bar{\varphi}=\varphi(p x) .
$$

Equation 34. The equation with varying transfer coefficient of power-law type

$$
u_{t}=a\left(u^{k} u_{x}\right)_{x}+b u^{-k}+f\left(u^{k+1}-w^{k+1}\right), \quad w=u(p x, t),
$$


admits the functional separable solution

$$
u(x, t)=[b(k+1) t+\varphi(x)]^{\frac{1}{k+1}},
$$

where the function $\varphi=\varphi(x)$ is described by the pantograph-type ODE:

$$
a \varphi_{x x}^{\prime \prime}+(k+1) f(\varphi-\bar{\varphi})=0, \quad \bar{\varphi}=\varphi(p x) .
$$

Equation 35. The equation with varying transfer coefficient of exponential type

$$
u_{t}=a\left(e^{\lambda u} u_{x}\right)_{x}+e^{\lambda u} f(u-w), \quad w=u(p x, t),
$$

admits the additive separable solution

$$
u(x, t)=-\frac{1}{\lambda} \ln t+\varphi(x),
$$

where the function $\varphi=\varphi(x)$ is described by the pantograph-type ODE:

$$
a\left(e^{\lambda \varphi} \varphi_{x}^{\prime}\right)_{x}^{\prime}+\frac{1}{\lambda}+e^{\lambda \varphi} f(\varphi-\bar{\varphi})=0, \quad \bar{\varphi}=\varphi(p x) .
$$

Equation 36. The equation with varying transfer coefficient of general form

$$
u_{t}=\left[f_{u}^{\prime}(u) u_{x}\right]_{x}+\frac{a}{f_{u}^{\prime}(u)}+g(f(u)-f(w)), \quad w=u(p x, t),
$$

admits the generalized traveling wave solution in implicit form

$$
f(u)=a t+\varphi(x),
$$

where the function $\varphi=\varphi(x)$ is described by the pantograph-type ODE:

$$
\varphi_{x x}^{\prime \prime}+g(\varphi-\bar{\varphi})=0, \quad \bar{\varphi}=\varphi(p x) .
$$

Remark 10. Equations (35)-(38) and their solutions can be substantially generalized if the pantograph term $w=u(p x, t)$ is replaced by $w=u(\xi(x), t)$, where $\xi(x)$ is an arbitrary function (the solutions are sought in the same form).

\section{Some Generalizations}

\subsection{Nonlinear Multi-Pantograph Type PDEs}

The equations and their solutions considered above can be generalized to the case of nonlinear multi-pantograph type PDEs of the form

$$
u_{t}=\left[g(u) u_{x}\right]_{x}+F\left(u, w_{1}, \ldots, w_{m}\right), \quad w_{j}=u\left(p_{j} x, q_{j} t\right), \quad j=1, \ldots, m .
$$

Two such equations with exact solutions are given below.

Equation 37. Consider the nonlinear multi-pantograph type PDE:

$$
u_{t}=a\left(u^{k} u_{x}\right)_{x}+u^{n} f\left(w_{1} / u, \ldots, w_{m} / u\right), \quad w_{j}=u\left(p_{j} x, q_{j} t\right),
$$

which generalizes Equation (9). For $n \neq 1$, Equation (39) admits a self-similar solution of the same form as Equation (9):

$$
u(x, t)=t^{\frac{1}{1-n}} U(z), \quad z=x t^{\frac{n-k-1}{2(1-n)}},
$$


where the function $U=U(z)$ satisfies the second-order multi-pantograph type ODE:

$$
\begin{aligned}
& \frac{1}{1-n} U+\frac{n-k-1}{2(1-n)} z U_{z}^{\prime}=a\left(U^{k} U_{z}^{\prime}\right)_{z}^{\prime}+U^{n} f\left(q_{1}^{\frac{1}{1-n}} W_{1} / U, \ldots, q_{m}^{\frac{1}{1-n}} W_{m} / U\right), \\
& W_{j}=U\left(s_{j} z\right), \quad s_{j}=p_{j} q_{j}^{\frac{n-k-1}{2(1-n)}}, j=1, \ldots, m .
\end{aligned}
$$

Equation 38. The nonlinear multi-pantograph type PDE, more general than Equation (34),

$$
u_{t}=a\left(u^{k} u_{x}\right)_{x}+u f\left(w_{1} / u, \ldots, w_{m} / u\right), \quad w_{j}=u\left(p_{j} x, t\right), \quad w_{j}=u\left(p_{j} x, t\right),
$$

admits a solution of the same form as Equation (34):

$$
u(x, t)=e^{2 \lambda t} U(z), \quad z=e^{-k \lambda t} x,
$$

where $\lambda$ is an arbitrary constant and the function $U=U(z)$ is determined from the multi-pantograph type ODE:

$$
2 \lambda U-k \lambda z U_{z}^{\prime}=a\left(U^{k} U_{z}^{\prime}\right)_{z}^{\prime}+U f\left(W_{1} / U, \ldots, W_{m} / U\right), \quad W_{j}=U\left(p_{j} z\right) .
$$

5.2. Nonlinear Pantograph-Type PDEs Containing Unknown Functions with Dilation or Contraction of Arguments in the Derivative

One can also consider more general than Equation (3) nonlinear pantograph-type reaction-diffusion equations of the form

$$
u_{t}=\left[G(u, w) u_{x}\right]_{x}+F(u, w) .
$$

Below are three examples of exact solutions to such equations.

Equation 39. Consider the pantograph-type diffusion equation with varying transfer coefficient

$$
u_{t}=\left[G(u, w) u_{x}\right]_{x}, \quad w=u(p x, q t) .
$$

$1^{\circ}$. In the general case, this equation admits the self-similar solution

$$
u(x, t)=U(z), \quad z=x t^{-1 / 2},
$$

where the function $U=U(z)$ is described by the nonlinear pantograph-type ODE:

$$
\left[G(U, W) U_{z}^{\prime}\right]_{z}^{\prime}+\frac{1}{2} z U_{z}^{\prime}=0, \quad W=U(s z), \quad s=p q^{-1 / 2} .
$$

$2^{\circ}$. For $q=p$, Equation (40) admits the traveling-wave solution

$$
u(x, y)=U(z), \quad z=k x-\lambda t,
$$

where $k$ and $\lambda$ are arbitrary constants and the function $U=U(z)$ is described by the first-order pantograph-type ODE:

$$
k^{2} G(U, W) U_{z}^{\prime}+\lambda U=C, \quad W=U(p z) ;
$$

$C$ is an arbitrary constant.

Equation 40. Consider now the equation

$$
u_{t}=a\left(e^{\lambda w} u_{x}\right)_{x}+e^{\mu u} f(u-w), \quad w=u(p x, q t),
$$


which is obtained from Equation (10) by replacing the function $e^{\lambda u}$ with $e^{\lambda w}$. For $\mu \neq 0$, this equation, like Equation (10), admits an exact solution of the form

$$
u(x, t)=U(z)-\frac{1}{\mu} \ln t, \quad z=x t^{\frac{\lambda-\mu}{2 \mu}},
$$

where the function $U=U(z)$ is described by the nonlinear pantograph-type ODE:

$$
\begin{aligned}
& \frac{\lambda-\mu}{2 \mu} z U_{z}^{\prime}-\frac{1}{\mu}=a q^{-\lambda / \mu}\left(e^{\lambda W} U_{z}^{\prime}\right)_{z}^{\prime}+e^{\mu U} f\left(U-W+\frac{1}{\mu} \ln q\right), \\
& W=U(s z), \quad s=p q^{\frac{\lambda-\mu}{2 \mu}} .
\end{aligned}
$$

Equation 41. The equation, more general than Equation (41),

$$
u_{t}=\left[e^{\lambda u} g(u-w) u_{x}\right]_{x}+e^{\mu u} f(u-w), \quad w=u(p x, q t),
$$

depending on two arbitrary functions $f(y)$ and $g(y)$ also has a solution of the form (42).

\subsection{Principle of Analogy of Solutions}

A fairly general method for constructing exact solutions of partial functional differential pantograph-type equations is based on the use of the following principle.

The principle of analogy of solutions. The structure of exact solutions of functional differential equations of the form

$$
\begin{aligned}
& F\left(x, t, u, u_{x}, u_{t}, u_{x x}, u_{x t}, u_{t t}, \ldots, w, w_{x}, w_{t}, w_{x x}, w_{x t}, w_{t t}, \ldots\right)=0, \\
& w=u(p x, q t),
\end{aligned}
$$

is often (but not always) determined by the structure of solutions of simpler PDEs:

$$
F\left(x, t, u, u_{x}, u_{t}, u_{x x}, u_{x t}, u_{t t}, \ldots, u, u_{x}, u_{t}, u_{x x}, u_{x t}, u_{t t}, \ldots\right)=0 .
$$

Equation (44) does not contain the unknown function with dilated or contracted arguments; it is obtained from (43) by formally replacing $w$ with $u$.

Most of the solutions to nonlinear pantograph-type reaction-diffusion equations described in this article have been obtained using the principle of analogy. Let us illustrate what has been said with specific examples.

Example 1. Consider the pantograph-type PDE with power-law nonlinearities

$$
u_{t}=a u_{x x}+b u^{m} w^{k}, \quad w=u(p x, q t) .
$$

Following the principle of analogy of solutions, we set $w=u$ in Equation (45). As a result, we arrive at the simpler PDE

$$
u_{t}=a u_{x x}+b u^{m+k} .
$$

This equation admits a self-similar solution of the form [94]:

$$
u(x, t)=t^{\frac{1}{1-m-k}} U(z), \quad z=x t^{-1 / 2}, \quad k \neq 1-m .
$$


Using the principle of analogy, we seek the solution of the pantograph-type PDE (45) also in the form (46). As a result, for the function $U=U(z)$ we get the nonlinear pantograph-type ODE:

$$
\begin{aligned}
& a U_{z z}^{\prime \prime}+\frac{1}{2} z U_{z}^{\prime}-\frac{1}{1-m-k} U+b q^{\frac{k}{1-m-k}} U^{m} W^{k}=0 \\
& W=U(s z), \quad s=p q^{-1 / 2} .
\end{aligned}
$$

Example 2. Let us now consider the pantograph-type PDE equation with exponential nonlinearities

$$
u_{t}=a u_{x x}+b e^{\mu u+\lambda w}, \quad w=u(p x, q t) .
$$

Following the principle of analogy of solutions, we set $w=u$ in Equation (47). As a result, we arrive at the simpler PDE

$$
u_{t}=a u_{x x}+b e^{(\mu+\lambda) u} .
$$

This equation admits an invariant solution of the form [94]:

$$
u(x, t)=U(z)-\frac{1}{\mu+\lambda} \ln t, \quad z=x t^{-1 / 2}, \quad \mu \neq-\lambda .
$$

Using the principle of analogy, we seek the solution of the pantograph-type PDE (47) in the form (48). As a result, for the function $U=U(z)$ we obtain the nonlinear pantograph-type ODE:

$$
\begin{aligned}
& a U_{z z}^{\prime \prime}+\frac{1}{2} z U_{z}^{\prime}+\frac{1}{\mu+\lambda}+b q^{-\frac{\lambda}{\mu+\lambda}} e^{\mu U+\lambda W}=0, \\
& W=U(s z), \quad s=p q^{-1 / 2} .
\end{aligned}
$$

Note that the principle of analogy of solutions can also be successfully applied to construct exact solutions of functional differential wave-type PDEs [118] and PDEs of higher orders that contain unknown functions with dilated or contracted arguments.

\section{Brief Conclusions}

For the first time, we have described exact solutions of various classes of nonlinear pantograph-type reaction-diffusion PDEs that, in addition to the unknown function $u=$ $u(x, t)$, also contain functions with dilated or contracted arguments of the form $u(p x, t)$, $u(x, q t)$, and $u(p x, q t)$, where $p$ and $q$ are the free scaling parameters. We have presented examples of nonlinear pantograph-type PDEs that admit traveling-wave solutions, selfsimilar solutions, additive and multiplicative separable solutions, functional separable solutions, and some other exact solutions. Special attention is paid to nonlinear pantographtype PDEs of a rather general form, which contain one or two arbitrary functions. A number of exact solutions of more complex nonlinear functional differential equations with varying delay, which arbitrarily depends on time or spatial coordinate, are described. We have formulated the principle of analogy of solutions that allows effectively constructing exact solutions of nonlinear pantograph-type PDEs. The presented exact solutions can be used as test problems to evaluate the accuracy of numerical and approximate analytical methods for solving the corresponding nonlinear initial-boundary value problems for PDEs with varying delay of pantograph type.

Author Contributions: Conceptualization and methodology, A.D.P. and V.G.S.; formal analysis and investigation, A.D.P. and V.G.S.; writing-original draft preparation, V.G.S.; writing-review and editing, A.D.P.; supervision, A.D.P. All authors have read and agreed to the published version of the manuscript.

Funding: The study was supported by the Ministry of Education and Science of the Russian Federation within the framework of the State Assignment (Registration No. AAAA-A20-120011690135-5) 
and was partially supported by the Russian Foundation for Basic Research (Projects No. 18-29-10025 and No. 18-29-03228).

Conflicts of Interest: The authors declares no conflict of interest.

\section{References}

1. Bellman, R.; Cooke, K.L. Differential-Difference Equations; Academic Press: New York, NY, USA, 1963; ISBN: 9780080955148.

2. Elsgolt's L.E.; Norkin, S.B. Introduction to the Theory and Application of Differential Equations With Deviating Arguments; Academic Press: New York, NY, USA, 1973; ISBN: 9780122377501.

3. Myshkis, A.D. Linear Differential Equations with Retarded Argument; Nauka: Moscow, Russia, 1972. (In Russian)

4. Wu, J. Theory and Applications of Partial Functional Differential Equations; Springer: New York, NY, USA, 1996; ISBN: 9780387947716.

5. Mei, M.; Lin, C.-K.; Lin, C.-T.; So, J.W.-H. Traveling wavefronts for time-delayed reaction-diffusion equation: (I) Local nonlinearity. J. Dif. Equat. 2009, 247, 495-510. [CrossRef]

6. Lv, G.; Wang, Z. Stability of traveling wave solutions to delayed evolution equation. J. Dyn. Control Syst. 2015, 21, 173-187. [CrossRef]

7. Polyanin, A.D.; Sorokin, V.G. Nonlinear delay reaction-diffusion equations: Traveling-wave solutions in elementary functions. Appl. Math. Lett. 2015, 46, 38-43. [CrossRef]

8. Meleshko, S.V.; Moyo, S. On the complete group classification of the reaction-diffusion equation with a delay. J. Math. Anal. Appl. 2008, 338, 448-466. [CrossRef]

9. Polyanin, A.D.; Zhurov, A.I. Exact solutions of linear and nonlinear differential-difference heat and diffusion equations with finite relaxation time. Int. J. Non-Linear Mech. 2013, 54, 115-126. [CrossRef]

10. Polyanin, A.D.; Zhurov, A.I. Functional constraints method for constructing exact solutions to delay reaction-diffusion equations and more complex nonlinear equations. Commun. Nonlinear Sci. Numer. Simul. 2014, 19, 417-430. [CrossRef]

11. Polyanin, A.D.; Zhurov, A.I. Exact separable solutions of delay reaction-diffusion equations and other nonlinear partial functionaldifferential equations, Commun. Nonlinear Sci. Numer. Simul. 2014, 19, 409-416. [CrossRef]

12. Polyanin, A.D.; Zhurov, A.I. New generalized and functional separable solutions to nonlinear delay reaction-diffusion equations. Int. J. Non-Linear Mech. 2014, 59, 16-22. [CrossRef]

13. Polyanin, A.D.; Zhurov, A.I. Nonlinear delay reaction-diffusion equations with varying transfer coefficients: Exact methods and new solutions. Appl. Math. Lett. 2014, 37, 43-48. [CrossRef]

14. Polyanin, A.D.; Zhurov, A.I. Non-linear instability and exact solutions to some delay reaction-diffusion systems. Int. J. Non-Linear Mech. 2014, 62, 33-40. [CrossRef]

15. Polyanin, A.D.; Zhurov, A.I. The functional constraints method: Application to non-linear delay reaction-diffusion equations with varying transfer coefficients. Int. J. Non-Linear Mech. 2014, 67, 267-277. [CrossRef]

16. Polyanin, A.D.; Zhurov, A.I. The generating equations method: Constructing exact solutions to delay reaction-diffusion systems and other non-linear coupled delay PDEs. Int. J. Non-Linear Mech. 2015, 71, 104-115. [CrossRef]

17. Polyanin, A.D. Generalized traveling-wave solutions of nonlinear reaction-diffusion equations with delay and variable coefficients. Appl. Math. Lett. 2019, 90, 49-53. [CrossRef]

18. Polyanin, A.D.; Sorokin, V.G. A method for constructing exact solutions of nonlinear delay PDEs. J. Math. Anal. Appl. 2021, 494, 124619. [CrossRef]

19. Polyanin, A.D.; Sorokin, V.G. Construction of exact solutions to nonlinear PDEs with delay using solutions of simpler PDEs without delay. Commun. Nonlinear Sci. Numer. Simul. 2021, 95, 105634. [CrossRef]

20. Polyanin, A.D.; Zhurov, A.I. Generalized and functional separable solutions to non-linear delay Klein-Gordon equations. Commun. Nonlinear Sci. Numer. Simul. 2014, 19, 2676-2689. [CrossRef]

21. Polyanin, A.D.; Sorokin, V.G.; Vyazmin, A.V. Exact solutions and qualitative features of nonlinear hyperbolic reaction-diffusion equations with delay. Theor. Found. Chem. Eng. 2015, 49, 622-635. [CrossRef]

22. Long F.-S.; Meleshko, S.V. On the complete group classification of the one-dimensional nonlinear Klein-Gordon equation with a delay. Math. Methods Appl. Sci. 2016, 39, 3255-3270. [CrossRef]

23. Long F.-S.; Meleshko, S.V. Symmetry analysis of the nonlinear two-dimensional Klein-Gordon equation with a time-varying delay. Math. Methods Appl. Sci. 2017, 40, 4658-4673. [CrossRef]

24. Polyanin, A.D.; Sorokin, V.G. New exact solutions of nonlinear wave type PDEs with delay. Appl. Math. Lett. 2020, 108, 106512. [CrossRef]

25. Polyanin, A.D.; Zhurov, A.I. Exact solutions of non-linear differential-difference equations of a viscous fluid with finite relaxation time. Int. J. Non-Linear Mech. 2013, 57, 116-122. [CrossRef]

26. Shampine L.F.; Thompson, S. Numerical Solutions of Delay Differential Equations. In Delay Differential Equations: Recent Advances and New Directions; Springer: New York, NY, USA, 2009; pp. 245-271; ISBN: 9780387855943.

27. Rihan, F.A. Computational methods for delay parabolic and time-fractional partial differential equations. Numer. Meth. Partial Differ. Equat. 2010, 26, 1556-1571. [CrossRef]

28. Schiesser, W.E. Time Delay ODE/PDE Models: Applications in Biomedical Science and Engineering; CRC Press: Boca Raton, FL, USA, 2020; ISBN: 9780367427979 
29. Jordan, P.M.; Dai, W.; Mickens, R.E. A note on the delayed heat equation: Instability with respect to initial data. Mech. Res. Comm. 2008, 35, 414-420. [CrossRef]

30. Ockendon, J.R.; Tayler, A.B. The dynamics of a current collection system for an electric locomotive. Proc. R. Soc. Lond. A. 1971, 332, 447-468. [CrossRef]

31. Hall, A.J.; Wake, G.C. A functional differential equation arising in the modelling of cell growth. J. Aust. Math. Soc. Ser. B, 1989, 30, 424-435. [CrossRef]

32. Hall, A.J.; Wake, G.C.; Gandar, P.W. Steady size distributions for cells in one dimensional plant tissues. J. Math. Biol. 1991, 30, 101-123. [CrossRef]

33. Derfel, G.; van Brunt, B.; Wake, G.C. A cell growth model revisited. Funct. Differ. Equat. 2012, 19, 71-81.

34. Zaidi, A.A.; van Brunt, B.; Wake G.C. Solutions to an advanced functional partial differential equation of the pantograph type. Proc. R. Soc. A. 2015, 471, 20140947. [CrossRef]

35. Efendiev, M.; van Brunt, B.; Wake, G.C.; Zaidi, A.A. A functional partial differential equation arising in a cell growth model with dispersion. Math. Meth. Appl. Sci. 2018, 41, 1541-1553. [CrossRef]

36. Ambartsumyan, V.A. On the fluctuation of the brightness of the Milky Way. Dokl. Akad. Nauk SSSR 1944, 44, $223-226$.

37. Dehghan, M.; Shakeri, F. The use of the decomposition procedure of Adomian for solving a delay differential equation arising in electrodynamics. Phys. Scr. 2008, 78, 065004. [CrossRef]

38. Ajello, W.G.; Freedman, H.I.; Wu, J. Analysis of a model representing stage-structured population growth with state-dependent time delay. SIAM J. Appl. Math. 1992, 52, 855-869. [CrossRef]

39. Mahler, K. On a special functional equation. J. Lond. Math. Soc. 1940, 1, 115-123. [CrossRef]

40. Ferguson, T.S. Lose a dollar or double your fortune. In Proceedings of the 6th Berkeley Symposium on Mathematical Statistics and Probability; Le Cam, L.M., Neyman, J., Scott, E.L., Eds.; University California Press: Berkeley, CA, USA, 1972; Volume III, pp. 657-666; ISBN: 9780520021853.

41. Robinson, R.W. Counting labeled acyclic digraphs. In New Directions in the Theory of Graphs; Harari, F., Ed.; Academic Press: New York, NY, USA, 1973; pp. 239-273; ISBN: 9780123242556.

42. Gaver, D.P. An absorption probablility problem. J. Math. Anal. Appl. 1964, 9, 384-393. [CrossRef]

43. Zhang, F.; Zhang, Y. State estimation of neural networks with both time-varying delays and norm-bounded parameter uncertainties via a delay decomposition approach. Commun. Nonlinear Sci. Numer. Simul. 2013, 18, 3517-3529. [CrossRef]

44. Fox, L.; Mayers, D.F.; Ockendon, J.R.; Tayler, A.B. On a functional differential equation. IMA J. Appl. Math. 1971, 8, 271-307. [CrossRef]

45. Iserles, A. On the generalized pantograph functional differential equation. Eur. J. Appl. Math. 1993, 4, 1-38. 00000966. [CrossRef]

46. Kate, T.; McLeod, J.B. Functional-differential equation $y^{\prime}=a y(\lambda t)+b y(t)$. Bull. Am. Math. Soc. 1971, 77, 891-937. [CrossRef]

47. Liu, M.Z.; Li, D. Properties of analytic solution and numerical solution of multi-pantograph equation. Appl. Math. Comput. 2004, 155, 853-871. [CrossRef]

48. Van Brunt, B.; Wake, G.C. A Mellin transform solution to a second-order pantograph equation with linear dispersion arising in a cell growth model. Eur. J. Appl. Math. 2011, 22, 151-168. [CrossRef]

49. Yüzbasi, S.; Sezer, M. An exponential approximation for solutions of generalized pantograph-delay differential equations. Appl. Math. Model. 2013, 37, 9160-9173. [CrossRef]

50. Reutskiy, S.Y. A new collocation method for approximate solution of the pantograph functional differential equations with proportional delay. Appl. Math. Comput. 2015, 266, 642-655. [CrossRef]

51. Isik, O.R.; Turkoglu, T. A rational approximate solution for generalized pantograph-delay differential equations. Math. Meth. Appl. Sci. 2016, 39, 2011-2024. [CrossRef]

52. Patade, J.; Bhalekar, S. Analytical solution of pantograph equation with incommensurate delay. Phys. Sci. Rev. 2017, 2, 20165103. [CrossRef]

53. Bahgat, M.S.M. Approximate analytical solution of the linear and nonlinear multi-pantograph delay differential equations. Phys. Scr. 2020, 95, 055219. [CrossRef]

54. Hou, C.-C.; Simos, T.E.; Famelis, I.T. Neural network solution of pantograph type differential equations. Math. Meth. Appl. Sci. 2020, 43, 3369-3374. [CrossRef]

55. Alrabaiah, H.; Ahmad, I.; Shah, K.; Rahman, G.U. Qualitative analysis of nonlinear coupled pantograph differential equations of fractional order with integral boundary conditions. Bound. Value Probl. 2020, 2020, 138. [CrossRef]

56. Liu, Y. On the $\theta$-method for delay differential equations with infinite lag. J. Comput. Appl. Math. 1996, 71, 177-190. [CrossRef]

57. Bellen, A.; Guglielmi, N.; Torelli, L. Asymptotic stability properties of $\theta$-methods for the pantograph equation. Appl. Numer. Math. 1997, 24, 279-293. [CrossRef]

58. Koto, T. Stability of Runge-Kutta methods for the generalized pantograph equation. Numer. Math. 1999, 84, 233-247. [CrossRef]

59. Bellen, A. Preservation of superconvergence in the numerical integration of delay differential equations with proportional delay. IMA J. Numer. Anal. 2002, 22, 529-536. [CrossRef]

60. Guglielmi, N.; Zennaro, M. Stability of one-leg $\theta$-methods for the variable coefficient pantograph equation on the quasi-geometric mesh. IMA J. Numer. Anal. 2003, 23 421-438. [CrossRef] 
61. Xu, Y.; Liu, M.Z. H-stability of Runge-Kutta methods with general variable stepsize for pantograph equation. Appl. Math. Comput. 2004, 148, 881-892. [CrossRef]

62. Evans, D.J.; Raslan, K.R. The Adomian decomposition method for solving delay differential equation. Int. J. Comput. Math. 2005, 82, 49-54. [CrossRef]

63. Li, D.; Liu, M.Z. Runge-Kutta methods for the multi-pantograph delay equation. Appl. Math. Comput. 2005, 163, 383-395. [CrossRef]

64. Liu, M.Z.; Yang, Z.W.; Xu, Y. The stability of modified Runge-Kutta methods for the pantograph equation. Math. Comput. 2006, 75, 1201-1215. [CrossRef]

65. Sezer, M.; Akyüz-Dascioglu, A. A Taylor method for numerical solution of generalized pantograph equations with linear functional argument. J. Comput. Appl. Math. 2007, 200, 217-225. [CrossRef]

66. Sezer, M.; Yalçinbaş, S.; Sahin, N. Approximate solution of multi-pantograph equation with variable coefficients. J. Comput. Appl. Math. 2008, 214, 406-416. [CrossRef]

67. Saadatmandi, A.; Dehghan, M. Variational iteration method for solving a generalized pantograph equation. Comput. Math. Appl. 2009, 58, 2190-2196. [CrossRef]

68. Brunner, H.; Huang, Q.; Xie, H. Discontinuous Galerkin methods for delay differential equations of pantograph type. SIAM J. Numer. Anal. 2010, 48, 1944-1967. [CrossRef]

69. Shakeri, F.; Dehghan, M. Application of the decomposition method of Adomian for solving the pantograph equation of order $m$. Z. Naturforsch. 2010, 65a, 453-460. [CrossRef]

70. Yusufoğlu, E. An efficient algorithm for solving generalized pantograph equations with linear functional argument. Appl. Math. Comput. 2010, 217, 3591-3595. [CrossRef]

71. Gülsu, M.; Sezer, M. A Taylor collocation method for solving high-order linear pantograph equations with linear functional argument. Numer. Meth. Partial Differ. Equat. 2011, 27, 1628-1638. [CrossRef]

72. Yalçinbaş, S.; Aynigül, M.; Sezer, M. A collocation method using Hermite polynomials for approximate solution of pantograph equations. J. Frankl. Inst. 2011, 348, 1128-1139. [CrossRef]

73. Yüzbaşi, S.; Şahin, N.; Sezer, M. A Bessel collocation method for numerical solution of generalized pantograph equations. Numer. Methods Partial Differ. Equ. 2011, 28, 1105-1123. [CrossRef]

74. Sedaghat, S.; Ordokhani, Y.; Dehghan, M. Numerical solution of the delay differential equations of pantograph type via Chebyshev polynomials. Commun. Nonlinear Sci. Numer. Simul. 2012, 17, 4815-4830. [CrossRef]

75. Tohidi, E.; Bhrawy, A.H.; Erfani, K. A collocation method based on Bernoulli operational matrix for numerical solution of generalized pantograph equation. Appl. Math. Model. 2013, 37, 4283-4294. [CrossRef]

76. Doha, E.H.; Bhrawy, A.H.; Baleanu, D.; Hafez, M. A new Jacobi rational-Gauss collocation method for numerical solution of generalized pantograph equations. Appl. Numer. Math. 2014, 77, 43-54. [CrossRef]

77. Wang, W. High order stable Runge-Kutta methods for nonlinear generalized pantograph equations on the geometric mesh. Appl. Math. Model. 2015, 39, 270-283. [CrossRef]

78. Wang, W. Fully-geometric mesh one-leg methods for the generalized pantograph equation: Approximating Lyapunov functional and asymptotic contractivity. Appl. Numer. Math. 2017, 117, 50-68. [CrossRef]

79. Yang, C. Modified Chebyshev collocation method for pantograph-type differential equations. Appl. Numer. Math. 2018, 134, 132-144. [CrossRef]

80. Yang, C.; Lv, X. Generalized Jacobi spectral Galerkin method for fractional pantograph differential equation. Math. Methods Appl. Sci. 2021, 44, 153-165. [CrossRef]

81. Li, X.Y.; Wu, B.Y. A continuous method for nonlocal functional differential equations with delayed or advanced arguments. J. Math. Anal. Appl. 2014, 409, 485-493. [CrossRef]

82. Liu, C.-S. Basic theory of a class of linear functional differential equations with multiplication delay. arXiv 2018, arXiv:1605.06734v4.

83. Rossovskii, L.E. Elliptic functionally-differential equations with contractions of arguments. Dokl. Math. 2006, 74, 809-811. [CrossRef]

84. Rossovskii, L.E. Elliptic functional differential equations with contractions and extensions of independent variables of the unknown function. J. Math. Sci. 2017, 223, 351-493. [CrossRef]

85. Skubachevskii, A.L. Boundary-value problems for elliptic functional-differential equations and their applications. Russ. Math. Surv. 2016, 71, 801-906. [CrossRef]

86. Abazari, R.; Ganji, M. Extended two-dimensional DTM and its application on nonlinear PDEs with proportional delay. Int. J. Comput. Math. 2011, 88, 1749-1762. [CrossRef]

87. Grover, D.; Sharma, D.; Singh, P. Accelerated HPSTM: An efficient semi-analytical technique for the solution of nonlinear PDE's Nonlinear Eng. 2020, 9, 329-337. [CrossRef]

88. Aksenov, A.V.; Polyanin, A.D. Methods for constructing complex solutions of nonlinear PDEs using simpler solutions. Mathematics 2021, 9, 345. [CrossRef]

89. Solodushkin, S.I.; Yumanova, I.F.; Staelen, R.D. First-order partial differential equations with time delay and retardation of a state variable. J. Comput. Appl. Math. 2015, 289, 322-330. [CrossRef] 
90. Sakar, M.G.; Uludag, F.; Erdogan, F. Numerical solution of time-fractional nonlinear PDEs with proportional delays by homotopy perturbation method. Appl. Math. Model. 2016, 40, 6639-6649. [CrossRef]

91. Bekela, A.S.; Belachew, M.T.; Wole, G.A. A numerical method using Laplace-like transform and variational theory for solving time-fractional nonlinear partial differential equations with proportional delay. Adv. Differ. Equat. 2020, 2020, 586. [CrossRef]

92. Tang, C.; Zhang, C. A fully discrete $\theta$-method for solving semi-linear reaction-diffusion equations with time-variable delay. Math. Comput. Simul. 2021, 179, 48-56. [CrossRef]

93. Polyanin, A.D.; Zaitsev, V.F. Handbook of Nonlinear Partial Differential Equations, 2nd ed.; CRC Press: Boca Raton, FL, USA, 2012; ISBN: 9781420087239.

94. Dorodnitsyn, V.A. On invariant solutions of the equation of non-linear heat conduction with a source. USSR Comput. Math. Math Phys. 1982, 22, 115-122. [CrossRef]

95. Nucci, M.C.; Clarkson, P.A. The nonclassical method is more general than the direct method for symmetry reductions. An example of the Fitzhugh-Nagumo equation. Phys. Lett. A 1992, 164, 49-56. [CrossRef]

96. Kudryashov, N.A. On exact solutions of families of Fisher equations. Theor. Math. Phys. 1993, 94, 211-218. [CrossRef]

97. Galaktionov, V.A. Quasilinear heat equations with first-order sign-invariants and new explicit solutions. Nonlinear Anal. Theor. Meth. Appl. 1994, 23, 1595-1621. [CrossRef]

98. Ibragimov, N.H. (Ed.) Vol. 1, Symmetries, Exact Solutions and Conservation Laws. In CRC Handbook of Lie Group Analysis of Differential Equations; CRC Press: Boca Raton, FL, USA, 1994; ISBN 9780849344886.

99. Doyle, P.W.; Vassiliou, P.J. Separation of variables for the 1-dimensional non-linear diffusion equation. Int. J. Non-Linear Mech. 1998, 33, 315-326. [CrossRef]

100. Pucci, E.; Saccomandi, G. Evolution equations, invariant surface conditions and functional separation of variables. Phys. D 2000, 139, 28-47. [CrossRef]

101. Estevez, P.G.; Qu, C.; Zhang, S. Separation of variables of a generalized porous medium equation with nonlinear source. J. Math. Anal. Appl. 2002, 275, 44-59. [CrossRef]

102. Kaptsov O.V.; Verevkin, I.V. Differential constraints and exact solutions of nonlinear diffusion equations. J. Phys. A Math. Gen. 2003, 36, 1401-1414. [CrossRef]

103. Galaktionov, V.A.; Svirshchevskii, S.R. Exact Solutions and Invariant Subspaces of Nonlinear Partial Differential Equations in Mechanics and Physics; Chapman \& Hall/CRC Press: Boca Raton, FL, USA, 2007; ISBN: 9781584886631.

104. Vaneeva, O.O.; Johnpillai, A.G.; Popovych, R.O.; Sophocleous, C. Extended group analysis of variable coefficient reactiondiffusion equations with power nonlinearities. J. Math. Anal. Appl. 2007, 330, 1363-1386. [CrossRef]

105. Vaneeva, O.O.; Popovych, R.O.; Sophocleous, C. Extended group analysis of variable coefficient reaction-diffusion equations with exponential nonlinearities. J. Math. Anal. Appl. 2012, 396, 225-242. [CrossRef]

106. Broadbridge, P.; Bradshaw-Hajek, B.H. Exact solutions for logistic reaction-diffusion equations in biology. Z. Angew. Math. Phys. 2016, 67, 93. [CrossRef]

107. Cherniha, R.; Serov, M.; Pliukhin, O. Nonlinear Reaction-Diffusion-Convection Equations: Lie and Conditional Symmetry, Exact Solutions and Their Applications; Chapman \& Hall/CRC Press: Boca Raton, FL, USA, 2018; ISBN: 9781498776196.

108. Bradshaw-Hajek, B.H. Nonclassical symmetry solutions for non-autonomous reaction-diffusion equations. Symmetry 2019, 11, 208. [CrossRef]

109. Goard, J.; Broadbridge, P. A note on separation of variables solutions of generalized nonlinear diffusion equations. Appl. Math. Lett. 2019, 98, 7-12. [CrossRef]

110. Kosov, A.A.; Semenov, E.I. The Lambert function and exact solutions of nonlinear parabolic equations. Russ. Math. 2019, 63, 10-16. [CrossRef]

111. Polyanin, A.D. Construction of exact solutions in implicit form for PDEs: New functional separable solutions of non-linear reaction-diffusion equations with variable coefficients. Int. J. Non-Linear Mech. 2019, 111, 95-105. [CrossRef]

112. Polyanin, A.D. Functional separable solutions of nonlinear reaction-diffusion equations with variable coefficients. Appl. Math. Comput. 2019, 347, 282-292. [CrossRef]

113. Polyanin, A.D. Comparison of the effectiveness of different methods for constructing exact solutions to nonlinear PDEs. Generalizations and new solutions. Mathematics 2019, 7, 386. [CrossRef]

114. Kosov, A.A.; Semenov, E.I. Exact solutions of the generalized Richards equation with power-law nonlinearities. Diff. Equ. 2020, 56, 1119-1129. [CrossRef]

115. Opanasenko, S.; Boyko, V.; Popovych, R.O. Enhanced group classification of nonlinear diffusion-reaction equations with gradient-dependent diffusivity. J. Math. Anal. Appl. 2020, 484, 123739. [CrossRef]

116. Polyanin, A.D. Functional separation of variables in nonlinear PDEs: General approach, new solutions of diffusion-type equations. Mathematics 2020, 8, 90. [CrossRef]

117. Polyanin, A.D.; Zhurov, A.I. Separation of variables in PDEs using nonlinear transformations: Applications to reaction-diffusion type equations. Appl. Math. Lett. 2020, 100, 106055. [CrossRef]

118. Polyanin, A.D.; Sorokin V.G. Exact solutions of nonlinear partial differential equations with pantograph type variable delay. Bull. NRNU MEPhI 2020, 9, 315-328. (In Russian) [CrossRef] 